\title{
Design and Development of Computer Aided Teaching Software for Mechanical Principle Course
}

\author{
YANG Yong a, ${ }^{\text {, }}$, JIANG Jing-liang, JIN Xia, LIU Xin-fu \\ School of Mechanical Engineering, Qingdao Technological University, \\ Qingdao 266520, Shandong, China \\ ayyong901@163.com
}

Keywords: Mechanical principle, Teaching software, Software development, Innovation design ability.

\begin{abstract}
For the current teaching of mechanical principle course, there is a lack of a set of applicative software which is helpful to carry out the design and optimization of mechanical products. To solve this problem, according to the actual requirements of mechanism analysis and project design during teaching, a set of computer aided teaching software which can carry out mechanism analysis and design optimization for linkage mechanism, cam mechanism and gear mechanism is developed, and the software function module design project is put forward too. Practical application shows that the teaching software can simulate and show the new scheme of mechanical product proposed by students and make the students get a deeper understanding of the theory, so as to effectively cultivate the students' ability in innovative design and product development
\end{abstract}

\section{《机械原理》计算机辅助教学软件设计与开发}

\author{
杨勇a, ${ }^{*}$, 江京亮，金霞，刘新福
}

青岛理工大学机械工程学院, 青岛, 山东, 中国

ayyong901@163.com

关键词: 机械原理; 教学软件; 软件开发; 创新设计能力

中文摘要: 当前《机械原理》课程教学中, 缺乏一套适用的软件辅助进行机械产品方案的设 计和优化。为解决该问题, 从《机械原理》课程机构分析与方案设计教学的实际要求出发, 开发了一套能完成连杆机构、凸轮机构和齿轮机构等典型机构分析与设计优化功能的教学辅 助软件, 提出了该软件的功能模块设计方案。实践应用表明, 该教学软件能够将学生提出的 新方案在计算机中预演, 加深学生对理论知识的理解, 有效培养学生的创新设计能力和产品开发 能力。

\section{1. 引言}

2013年6月, 我国加入《华盛顿协议》，表明我国工程教育的质量得到了国际社会的认可， 而同时该协议也对我国工程教育提出了新要求, 要把培养工程师的能力作为首要目标。美国 工程与技术鉴定委员会（ABET）制定了工程准则2000（EAC200），提出了11条工程人才评 估标准, 强调了适用于工程教育的基于产出的核心思想（Outcome-based system）。它的精华 在于把焦点放在学生 “学到了什么”, 强调了毕业生实际能力, 而不像以往强调的是学校 “教 了什么”。针对工程教育, 我国进行了工程教育专业认证试点工作和卓越工程师教育培养计 
划等改革工作，前者要求高校能够培养在知识、能力和素质方面培养具有一定竞争优势的工 程技术人才, 而后者强化培养学生的工程能力和创新能力, 造就一大批创新能力强、适应经 济社会发展需要的高质量各类型工程技术人才 ${ }^{[1-2]}$ 。以上国内外工程教育的发展和改革都表 明：培养学生的创新能力是新时期工程教育改革的主要方向, 能力的达成已成为当前高校工 程教育改革发展的核心和首要目标。

《机械原理》课程作为高等院校机械类专业的一门主干技术基础课程，根据其课程的性 质、地位和内容, 即它使学生掌握有关机构学和机械动力学的基本理论、基本知识和基本技 能，使之初步具有拟定机械运动方案、分析和设计机构的能力，因而在培养机械类高级工程 技术人才的全局中具有增强学生对机械技术工作的运作能力和开发创造能力的作用，及其在 创新设计的知识结构中占有的核心地位, 决定了《机械原理》课程在培养学生创新能力方面 起着举足轻重的作用。

当前，有关《机械原理》课程的改革主要集中在课程体系、教学模式、教学方法以及考核 方式等方面 ${ }^{[3-5]}$, 而有关《机械原理》课程的辅助教学软件很少有报道, 特别是培养和提高学 生创新设计能力的辅助教学软件系统更是缺乏。鉴于该现状, 本论文从《机械原理》课程教 学实际需求出发, 开发出一套对机构进行结构分析与运动过程仿真的教学辅助软件, 实现对 连杆机构、凸轮机构和齿轮机构等典型机构的结构分析与优化设计, 从而加深学生对理论知 识的理解，培养和提高学生的创新设计能力。

\section{2. 软件功能模块设计}

《机械原理》辅助教学软件功能模块包括三部分: (1)系统管理模块。该模块是为用户的 软件进行保护验证, 以防不测; (2)设计计算及演示模块。该工具包含对机构的设计计算、对 机构运动过程中产生的位移、速度、加速度的计算及曲线输出分析、以及后续的力、力矩、 扭矩的分析, 演示模块。其中连杆机构模块完成曲柄滑块机构的解析法设计计算及机构运动、 从动件位移、速度、加速度的动态演示; 凸轮机构中分为圆柱凸轮和盘形凸轮分别设计; 齿 轮传动分为直齿轮、斜齿轮、锥齿轮和人字齿等部分, 蜗轮蜗杆机构相对比较特殊, 单列为 蜗杆部分和蜗轮部分; 除了这些常见的机构, 还有一些其他的机构, 如棘轮机构、槽轮机构、 螺纹机构和万向铰链机构。(3)在线帮助模块。主要包括两方面内容: (1)典型机构分析和设计 问题的数学模型的构造; (2)将所构造的数学模型转化为计算机可实现的程序, 以及对程序的 流程合理性、结构简洁性、可读性和维护性等方面的要求。

其中设计计算及演示模块是该软件的主要功能模块，包含数据输入、后台计算、数据结 果输出、动态图形结果输出、数据曲线输出及结果保存等几个部分。数据输入设计为对话框 形式, 通过Text等控件获得输入数据; 使用MSFlex-Grid控件创建数据表格, 并将数据计算结 果写入表格的对应行和列, 得到以数值形式表示的从动件位移、速度、加速度等; 使用point、 line等方法、circle等函数及shape、picture box等控件, 实现动态图形结果演示及运动参数曲线 演示功能; 程序设计过程中，同时给出了不同的错误提示信息。

设计计算及演示模块实现过程如下:

第一步，定义从动件位移、从动件速度、从动件加速度、机架长、计算步长、点数和间 隔等变量; 第二步, 加载模块时初始化控件, 建立连杆机构、凸轮机构齿轮机构等功能窗体, 在窗体中依次加载参数输入，计算结果、图形显示选项卡; 第三步，处理多页面窗体，并在 各选项卡中加载label、Text、command、Timer等控件。在参数输入选项卡中给定设计已知条 件, 为计算结果列表中各标题赋值, 定义图形显示坐标、绘图比例; 第四步, 调用计算过 程子程序, 实现机构的解析法设计计算, 并将计算结果显示于计算结果选项卡; 第五步, 将 计算结果存为文本文件并打印; 第六步, 调用相应模块, 比如定义坐标位置子程序、求图形 最大值子程序; 第七步, 调用动态绘图子程序, 利用解析解的精确结果作图, Timer计时器实 现机构运动动态演示及运动参数变化曲线的动态变化过程。 


\section{3. 辅助教学软件设计与开发}

\section{1 登录界面及主菜单窗口的设计}

首先在新建工程内新建窗体, 选择对话框形式的窗体。登录界面的制作就在这个窗体内 完成, 第一步, 在整个窗口界面内插入Image1, 同时在Image1中间位置插入一个Frame控件, 提供给用户的一些信息及登录按钮可在Frame控件里显示出来。第二步, 在Frame控件里插入 文本，输入显示给用户的信息，同时插入登录按钮。登录界面即可完成，效果图如图1所示。 其次制作主菜单窗口, 在所在工程内新建窗体, 选择窗体形式, 整个菜单的主界面就在这个 窗口里完成。这个部分可分为三个步骤完成, 第一步, 点击菜单编辑器, 把所需要的主菜单 及子菜单输入进去即可完成主菜单的编辑。该过程中的所有机构及用途在上面已作简单介绍。 第二步, 编辑工具栏, 在主菜单下面一栏插入一系列Command控件, 然后在属性栏插入所需 图标即可。第三步, 快速构建机构工具栏的编辑, 在界面右侧插入一个Frame控件, 在Frame 控件里可插入相应的图标及文本, 然后对这些图标及文本进行有序的排列即可。主菜单界面 的效果图如图2所示:

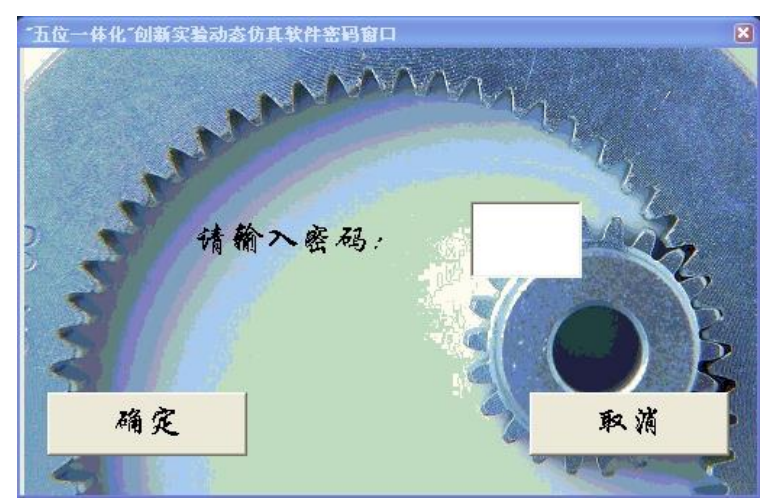

图1 软件登录界面设计

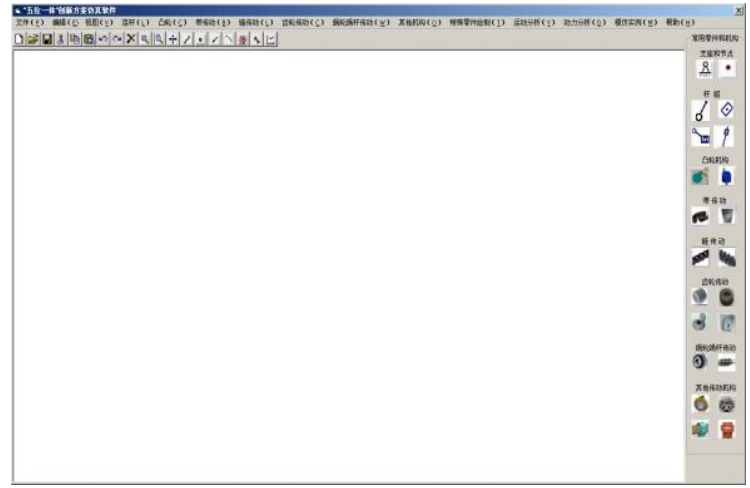

图2 软件主菜单窗口设计

\section{2 登录界面及主菜单窗口的设计}

软件的主要功能实现还需要一个对话框来实现人机信息交流。新建对话框并插入参数输 入所需要的Label和Text控件, 以及上传设计所需要的图片, 然后进行有序的排列。为了实现 对话框与主菜单的连接, 还需插入一个Command控件。所设计的曲柄滑块机构参数输入对话 框如图3所示，凸轮机构参数输入对话框设计如图4所示:

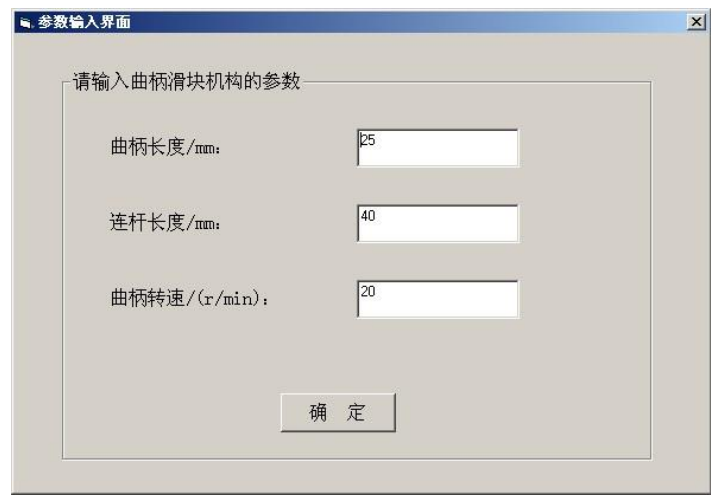

图3 曲柄滑块机构参数输入对话框设计

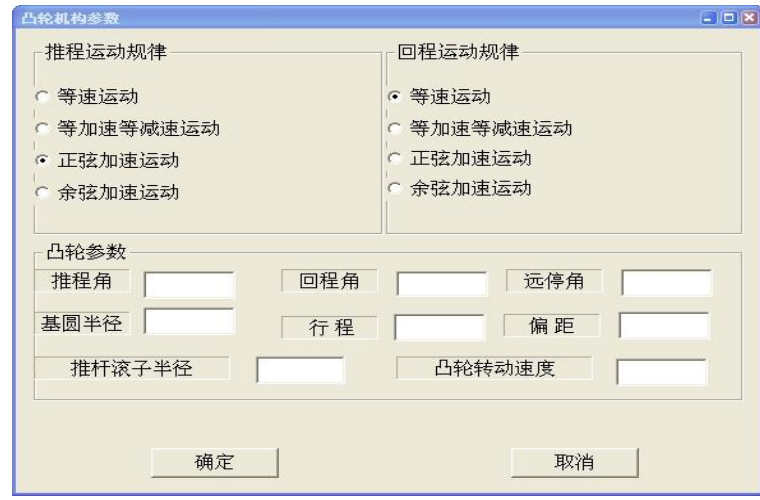

图4 凸轮机构参数输入对话框设计

停止、继续和清除等功能。通过相应参数的输入即可得到相应机构及其运动仿真分析过程。

下面以曲柄滑块机构运动仿真过程为例描述软件的运行过程。

单击控制窗口中的停止、继续、清除可以控制曲柄滑块机构的运动状况。此时点击菜单 栏中的运动分析，选择位移分析、速度分析，即可得相应的曲线分析图，如图5-6所示: 


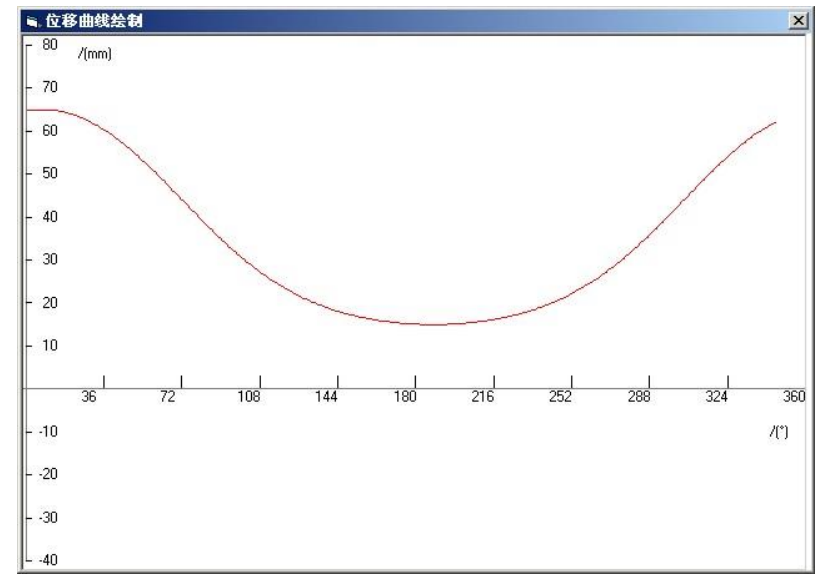

图5 位移分析曲线

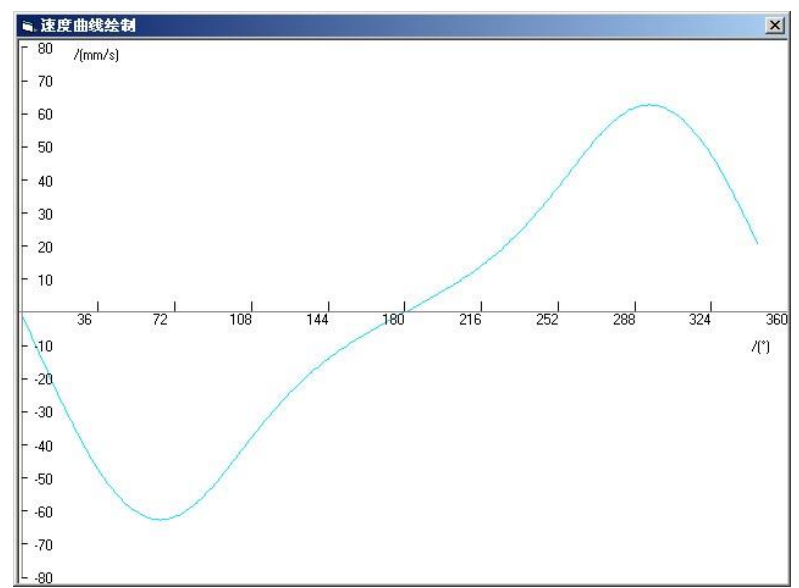

图6 速度分析曲线

\section{4. 结论}

(1) 开发了《机械原理》辅助教学软件, 针对曲柄滑块典型机构, 进行了参数化机构设 计与运动过程仿真分析, 获得了位移分析曲线、速度分析曲线。实例演示表明该辅助教学软 件能够对典型机构进行参数化设计，并进行运动过程仿真及其相应运动学物理量分析。

(2) 以往《机械原理》课程教学改革主要集中在课程体系、教学模式、教学方法以及考 核方式等方面, 而缺少教学辅助软件的开发。本软件系统的开发, 将是对《机械原理》课程 教学改革的有益补充, 使课程改革更加全面, 课程体系更加科学和系统化。

\section{致谢}

本文为山东省高等教育名校建设工程教改重点项目《以能力为核心重构《机械原理》课 程教学体系的综合改革与实践》（项目编号MX4-003）的阶段性成果之一。

\section{References}

[1] J. Wu, Ministry of Education to Start and Implement the Program of "A Plan for Educating and Training Outstanding Engineers", Teaching and Research Trends, vol.7, pp. 8, 2010.

[2] J. Lin, On Professional Training Program of "A Plan for Educating and Training Outstanding Engineers", Engineering Education Research of Tsinghua University, vol.2, pp. 4, 2011.

[3] W. D. Guo, R. Liu, and J.T. Li, Reform and Practice of the Teaching Method and Means of Theory of Machines and Mechanisms Course, Journal of Taiyuan University of Technology (SOCIAL SCIENCE EDITION), vol.26, pp. 57-60, 2008.

[4] H. Y. Yu, Reform and practice of mechanical principle course design, School education of China, vol.2, pp. 36, 2009.

[5] H. Li, Q.M. Zhang, and C.Z. Wang, Study of diversified and comprehensive examination way for mechanical principle course, Journal of Jimei University(Education Science Edition), vol.8, pp. 86-88, 2007. 